\title{
Estimation of the number of extreme pathways for metabolic networks
}

\author{
Matthew Yeung1, Ines Thiele ${ }^{1,2}$ and Bernard $\varnothing$ Palsson*1
}

Address: ${ }^{1}$ Dept. of Bioengineering, University of California, San Diego, 9500 Gilman Drive, La Jolla, CA 92093-0412, USA and 2Program in Bioinformatics, University of California, San Diego, 9500 Gilman Drive, La Jolla, CA 92093-0412, USA

Email: Matthew Yeung - myeung@bioeng.ucsd.edu; Ines Thiele - ithiele@bioeng.ucsd.edu; Bernard Ø Palsson* - palsson@ucsd.edu

* Corresponding author

Published: 27 September 2007

BMC Bioinformatics 2007, 8:363 doi:10.1/86/147/-2105-8-363
Received: 21 February 2007

Accepted: 27 September 2007

This article is available from: http://www.biomedcentral.com/I47I-2/05/8/363

(C) 2007 Yeung et al.; licensee BioMed Central Ltd.

This is an Open Access article distributed under the terms of the Creative Commons Attribution License (http://creativecommons.org/licenses/by/2.0), which permits unrestricted use, distribution, and reproduction in any medium, provided the original work is properly cited.

\begin{abstract}
Background: The set of extreme pathways $(\mathrm{ExPa}),\left\{\mathbf{p}_{i}\right\}$, defines the convex basis vectors used for the mathematical characterization of the null space of the stoichiometric matrix for biochemical reaction networks. ExPa analysis has been used for a number of studies to determine properties of metabolic networks as well as to obtain insight into their physiological and functional states in silico. However, the number of ExPas, $p=\left|\left\{\mathbf{p}_{i}\right\}\right|$, grows with the size and complexity of the network being studied, and this poses a computational challenge. For this study, we investigated the relationship between the number of extreme pathways and simple network properties.
\end{abstract}

Results: We established an estimating function for the number of ExPas using these easily obtainable network measurements. In particular, it was found that log $[p]$ had an exponential relationship with $\log \left[\sum_{i=1}^{R} d_{-_{i}} d_{+_{i}} c_{i}\right]$, where $R=\left|R_{\text {eff }}\right|$ is the number of active reactions in a network, $d_{-_{i}}$ and $d_{+_{i}}$ the incoming and outgoing degrees of the reactions $r_{i} \in R_{\text {eff }}$ and $c_{i}$ the clustering coefficient for each active reaction.

Conclusion: This relationship typically gave an estimate of the number of extreme pathways to within a factor of 10 of the true number. Such a function providing an estimate for the total number of ExPas for a given system will enable researchers to decide whether ExPas analysis is an appropriate investigative tool.

\section{Background}

Extreme pathways (ExPas) of a metabolic network are the irreducible set of vectors that define the basis of the nullspace of the network's stoichiometric matrix. Every allowable solution to the flux balance equations of a reaction network in steady state, $\mathbf{S} \cdot v=\mathbf{0}$, can be represented as a non-negative linear combination of the extreme pathway vectors. ExPas are biochemically and thermodynamically feasible pathways that transform a selection of the given substrates to a selection of allowable products. ExPas have been extensively used for the analyses of metabolic networks (see, for example, [1-5]). Typically, such analyses used ExPas to define possible phenotypic states of metabolic networks under different simulation conditions, to identify network redundancy, and to reveal eigenpathways that effectively characterize all relevant physiological 
states of a metabolic network. Modified versions of ExPa analyses have also been applied to regulatory networks $[6,7]$ and signaling pathways [8]. Such applications are still in their infancy and are important research topics. However, as the size of a network increases, the redundancy of the network, that is, the number of different pathways that transform given substrate(s) to given product(s) $[9,10]$, becomes more apparent, and the number of ExPas increases rapidly. Redundancy also exists in small systems but this can be easily handled and even provide insights to legitimate alternative pathways. As the number of ExPas increases at a drastic rate, performing insightful analyses using ExPas become increasingly difficult.

The fact that the set of ExPas of a biochemical reaction network defines the boundaries of its convex steady-state solution space makes them a valuable tool for metabolic network analysis. Further, they emphasize alternative pathways that exist in a network, which may otherwise be overlooked, and that can enrich the understanding of its possible physiological states. However, the increasing details included in reconstructed metabolic networks lead to the combinatorial explosion of the number of ExPas and their computation time. A method providing a good estimate for the final number of ExPas for a given system will enable researchers to decide whether ExPas analysis is a appropriate tool for their objectives.

Another method often used for characterizing the steadystate solution space for a biochemical reaction network is known as Elementary Modes (EMs) [11]. Both ExPa and $\mathrm{EM}$ analyses require the resulting solution vectors to be non-decomposable and unique. In addition, ExPa vectors are required to be systemically independent [12]. As a result, ExPas for a system are a minimal set of EMs, and the number of ExPas is less than or equal to the number of EMs. Since both ExPas and EMs are biochemically and thermodynamically feasible pathways, the number of these pathways cannot be estimated using traditional graph theoretical algorithms, such as the Dijkstra's algorithm [13], for finding all shortest paths.

The combinatorial complexity of Elementary Modes of a network was previously described by Klamt et al. [14] by providing an upper-bound for the number of EMs. In their work, the authors considered the following combinatoric problem: given a network with $n$ reactions and $m$ metabolites, the maximal number of independent pathways occurs when each possible subset of the reactions consisting of the $m$ metabolites are independent. This maximal number was found to be $\left(\begin{array}{c}n \\ m+1\end{array}\right)$. They further improved the upper-bound by removing those reactions that were not utilized (redundant reactions) in the condition-specific models of the network. Klamt et al. generated 5 models of the E. coli reconstruction, which yielded 599 to 507632 elementary modes. The upper-bounds for these models ranged from $5.57 \times 10^{17}$ to $4.39 \times 10^{21}$, which were subsequently reduced to $1.67 \times 10^{11}$ to $4.85 \times 10^{13}$ after removing redundant reactions identified using FluxAnalyzer. Despite an improvement of a factor of 6 to 8 , when comparing the upper-bounds to the actual number of EMs calculated for the models, they typically overestimated by approximately $6 \times 109 \%$ [14]. Although their work dealt with EMs, the problem was constructed as a purely combinatoric problem. Therefore the same reasoning can be directly applied to Extreme Pathways.

In this study, we investigated the relationship between the number of ExPas for a given network, $p=\left|\left\{\mathbf{p}_{i}\right\}\right|$, and its basic network measurements. Several network measurements are commonly used in describing the topological features of a network and include connectivity, clustering coefficient, network diameter, and degree distribution [15]. The number of ExPas for a network can vary dramatically under different simulation conditions, that is, different environmental constraints. Consequently, basic network information such as the numbers of reactions and metabolites of the network cannot be solely used to provide a meaningful estimate. Since ExPas are connected reactions, we hypothesized that the higher the reaction connections, the larger the number of ExPas. Based on this hypothesis, we demonstrated an exponential relationship between $\log [p]$ and $\log \left[\sum_{i=1}^{R} d_{-_{i}} d_{+_{i}} c_{i}\right]$, where $R=\left|R_{\text {eff }}\right|$ is the active (or effective) reactions in the network, $d_{-i}$ and $d_{+_{i}}$ are the incoming and outgoing degrees of a reaction, and $c_{i}$ is the clustering coefficient for each active reaction. This relationship typically gave an estimate of the number of ExPas to within a factor of 10. Since these network measurements can be calculated quickly and easily for any sized network, an estimation of ExPa numbers can be readily obtained as this serves as an assessment of the feasibility of ExPas as an analysis tool.

\section{Results}

The number of extreme pathways (ExPa), $p=\left|\left\{\mathbf{p}_{i}\right\}\right|$, for a metabolic network increases drastically with the complexity and size of the network. An estimate for $p$ for a given network can help one decide whether ExPa analysis is a feasible tool for one's research objective. In this study, we 
investigated the relationship between $p$ and a number of factors, $\theta_{i}$, formed by simple network measurements such as the incoming and outgoing degree of reactions, $d\left(r_{i}\right)=$ $d_{\mp_{i}}$, and clustering coefficient of each reaction, $c\left(r_{i}\right)=c_{i}$. These measurements were chosen as they could be calculated quickly and easily, and their definitions can be found in the sections titled 'Reaction Connectivity $d_{ \pm}\left(r_{i}\right)=$ $d_{ \pm_{i}}$ ' and 'Reaction Clustering Coefficient $c_{i}=c\left(r_{i}\right)$ ', respectively. A total of 52 models, generated from 6 reconstructed metabolic networks by altering environmental conditions, were used to determine a relationship between $p$ and $\theta_{i}$. The ExPas and corresponding network measurements for these models were calculated and used to identify possible estimating functions. Analyses on logged data (the logarithmic values of the data) revealed an exponential relationship between $\log [p]$ and $\log \left[\theta_{i}\right]$. A total of 4 estimating functions using two factors were obtained, which were then tested for robustness using an additional 16 models. The numbers of ExPas for most of these models were successfully estimated to within a factor of 10 . We concluded that it was possible to formulate an estimating function for the number of ExPas of a model, $p^{\prime}$, that typically falls within a factor of 10 of the actual number of ExPa.

\section{Identification of Significant Contributing Factors}

We aimed to identify factors that can be used for establishing appropriate estimating functions. Desirable factors must be i) easily obtained and ii) specific for a given model. For example, network measurements such as the incoming and outgoing degrees of each reaction, $d\left(r_{i}\right)=$ $d_{\mp_{i}}$, their clustering coefficients, $c\left(r_{i}\right)=c_{i^{\prime}}$ as well as the number of external metabolites and their degrees can be obtained quickly. In addition, we required factors to be highly correlated to the number of ExPas and, furthermore, increased in values consistently with $p$ to avoid misrepresentation due to incomparable ranges. The network measurements used in this study are detailed in the 'Network Measurements' section.

A number of potential factors for the estimating functions were formed using the aforementioned network measurements (Table 1). The correlations of these potential factors and $p$ were evaluated using the Pearson's productmoment correlation, $r$, and Spearman-rank correlation, $\rho$. The Pearson's correlation is generally used as an indicator for the strength and direction of a linear relationship and is considered to be robust enough to handle non-parametric data. On the other hand, the Spearman's correlation describes the monotonic relationship between two variables without making any assumptions about the frequency distribution of the variables. We used both of these correlation coefficients on the original and logged data to avoid misinterpretation due to the wide ranges of data (Table 1). For the original data, the factor with the highest Pearson correlation before was found for $\theta_{1}=\sum_{i=1}^{R} d_{-_{i}} d_{+_{i}} c_{i}$, where $R=\left|R_{e f f}\right|$ is the number of active reactions in the network. In contrast, after data-logging, the strongest correlation with $p$ was found for $\theta_{2}=\sum_{i=1}^{R} d_{-i} d_{+_{i}}$. The correlation between these two factors themselves were extremely high ( 0.992176). In addition, both factors had the second highest Spearman correlations with $p$. Furthermore, these two factors also had ranges comparable to that of the number of ExPas.

Table I: Identification of Potential Contributing Factors

\begin{tabular}{|c|c|c|c|c|c|c|c|c|c|c|c|}
\hline & & $R$ & $* \sum\left(d_{+} d_{-}\right)$ & $\sum c$ & $* \sum\left(d_{+} d_{-} c\right)$ & $\frac{\sum\left(d_{+} d_{-}\right)}{R}$ & $\frac{\sum c_{i}}{R}$ & $\frac{\sum\left(d_{+} d_{-} c\right)}{R}$ & $\sum_{I} d_{i}$ & $\sum_{O} d$ & $\left(\sum_{I} d\right)\left(\sum_{O} d\right)$ \\
\hline \multirow{2}{*}{\multicolumn{2}{|c|}{$\begin{array}{l}r \text { Pre-log } \\
r \text { Post-log }\end{array}$}} & 0.459 & 0.656 & 0.509 & 0.666 & 0.6 & 0.0178 & 0.62 & 0.135 & 0.604 & 0.408 \\
\hline & & 0.827 & 0.875 & 0.764 & 0.870 & 0.860 & 0.343 & 0.856 & 0.426 & 0.561 & 0.623 \\
\hline \multicolumn{2}{|c|}{$\rho$} & 0.841 & 0.876 & 0.943 & 0.876 & 0.855 & -0.059 & 0.845 & 0.478 & 0.496 & 0.603 \\
\hline \multirow[t]{2}{*}{ Range } & Min & 8 & 15 & 5 & 2 & 1.67 & 0.09 & 0.06 & 2 & 2 & 6 \\
\hline & Max & 174 & 89132 & 35.08 & 5781.73 & 665.38 & 0.9 & 47.78 & 25 & 47 & 414 \\
\hline
\end{tabular}

A good factor for an estimating function must have a high correlation to that is being estimated. We further required that the factor must grow consistently with the number of ExPas. The rows labelled 'Pre-' and 'Post-' Log show the Pearson's correlations, $r$, between the number of ExPas and the corresponding factors. These factors were created using the following basic networks measurements: $R=R_{\text {eff }}$ the number of active reactions given the environmental conditions, $d_{ \pm}=d_{ \pm}\left(r_{i}\right)$ the incoming/outgoing connectivity of reaction $r_{i}, c=c\left(r_{i}\right)$ the clustering coefficient of the $i^{\text {th }}$ reaction, $I$ the set of input reactions, and $O$ the set of output reactions. Both Pearson's and Spearman's Rank correlation coefficients, $r$ and $\rho$ were used a guide to identify reliable contributing factors. Given this information, the final chosen factors are emphasized by an asterisk (*). 
Despite the fact that the factors $\theta_{1}$ and $\theta_{2}$ are so closely correlated, both of these factors were used to create estimates utilizing single factors in the following section.

\section{Single Factor Estimate}

The factors identified in the previous section, namely $\theta_{1}=\sum_{i=1}^{R} d_{-_{i}} d_{+_{i}} c_{i}$ and $\theta_{2}=\sum_{i=1}^{R} d_{-_{i}} d_{+_{i}}$ were used to formulate estimating functions for the number of extreme pathways, $p$. Preliminary analyses showed that the relationships between $\log [p]$ and $\log \left[\theta_{j}\right], j=1,2$, were nonlinear (Figure 1 and Figure 2). In particular, the expression found to best describe these relationships had the form

$$
\begin{aligned}
\log \left[p_{k}\right]=f_{i}\left(\theta_{j, k}\right) & =\alpha_{i, j}+\beta_{i, j} 10^{\gamma_{i, j}\left(\omega_{i} \log [R]+\log \left[\theta_{j, k}\right]\right)} \\
& =\alpha_{i, j}+\beta_{i, j}\left(R_{k}^{\omega_{i}} \theta_{j, k}\right)^{\gamma_{i, j}}, \quad i=1,2,
\end{aligned}
$$

where $\hat{p}_{k}$ is the estimated number of ExPas for a given model $k, \theta_{j, k}$ are the values of the factors $\theta_{j^{\prime}} j=1,2$ for the $k^{\text {th }}$ model, and $R_{k}$ is the number of active reactions in model $k$. The estimating functions were formulated using the factors $\theta_{j}$ solely (case $i=1$ ) and by scaling these factors with $R_{k}^{\omega}$ (case $\left.i=2\right)$. For the former, the parameter $\omega_{1}$ had the value 0 . For the latter case, $\omega_{2}$ was the optimal value

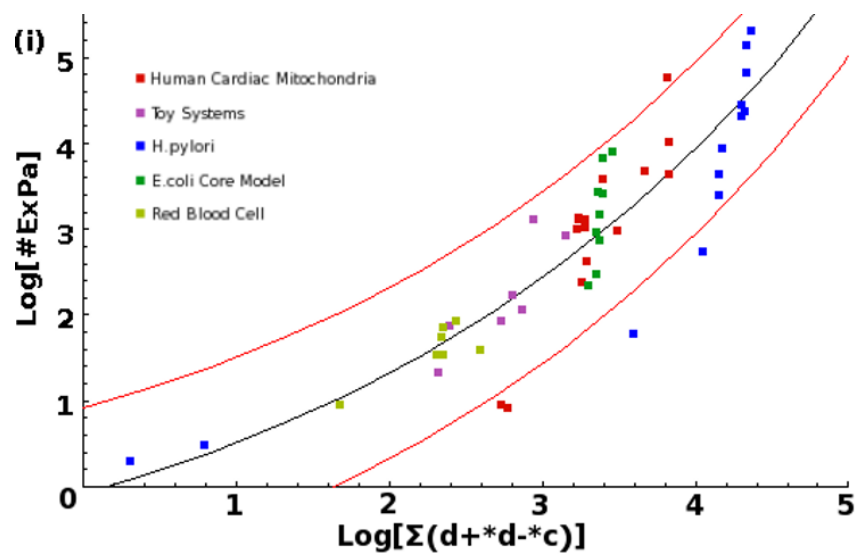

for which the highest Pearson's correlation between log $[p]$ and $\log \left[\hat{p}_{k}\right]$ could be obtained using the two different factors. This number was found to be the same for both factors and had the value $\omega_{2}=2.124857$. The parameters $\alpha_{i, j}, \beta_{i, j}$ and $\gamma_{i, j}$ take on different values for the four estimating functions $f_{i}\left(\theta_{j}\right)$, which are detailed in the following subsections 'Estimation Using $\theta_{1}$ ' and 'Estimation Using $\theta_{2}^{\prime}$.

\section{Estimation Using $\theta_{1}$}

Using factor $\theta_{1}=\sum_{i=1}^{R} d_{-i} d_{+_{i}} c_{i}$, the following estimating function was obtained when $\omega_{1}$ was applied:

$$
f_{1}\left(\theta_{1, k}\right)=-1.708123+1.624207\left(\sum_{i=1}^{R_{k}} d_{-_{i}} d_{+_{i}} c_{i}\right)^{0.135324}
$$

and with $\omega_{2}$ :

$$
f_{2}\left(\theta_{1, k}\right)=-0.750588+0.514844\left(R_{k}^{2.124857} \sum_{i=1}^{R} d_{-_{i}} d_{+_{i}} c_{i}\right)^{0.114214} .
$$

The fitted curves given by Equations (2) and (3) are shown in Figure 1. The Pearson's correlation was 0.883439 for the function given by Equation (2), whereas

\section{Figure I}

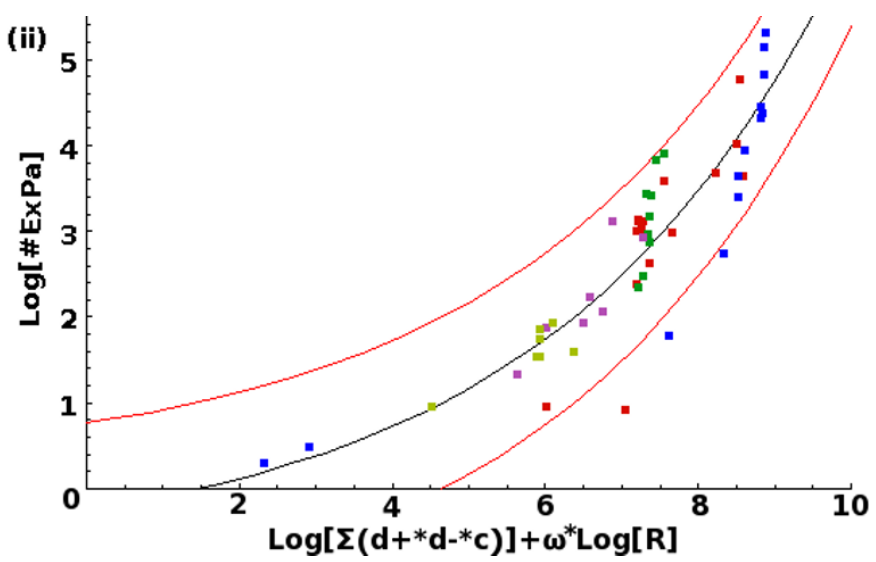

Relationship between the Number of ExPas and Factor $\theta_{1}=\sum_{i=1}^{R} d_{-i} d_{+_{i}} c_{i}$. Graphs displaying the two relationships derived from the factor $\theta_{1}=\sum_{i=1}^{R} d_{-_{i}} d_{+_{i}} c_{i}$. It was observed that $\theta_{1}$ had an exponential relation to $p$ as shown in (i). The use of $R^{\omega}$ as a scaling factor was found to improve the fit of the data (ii). 

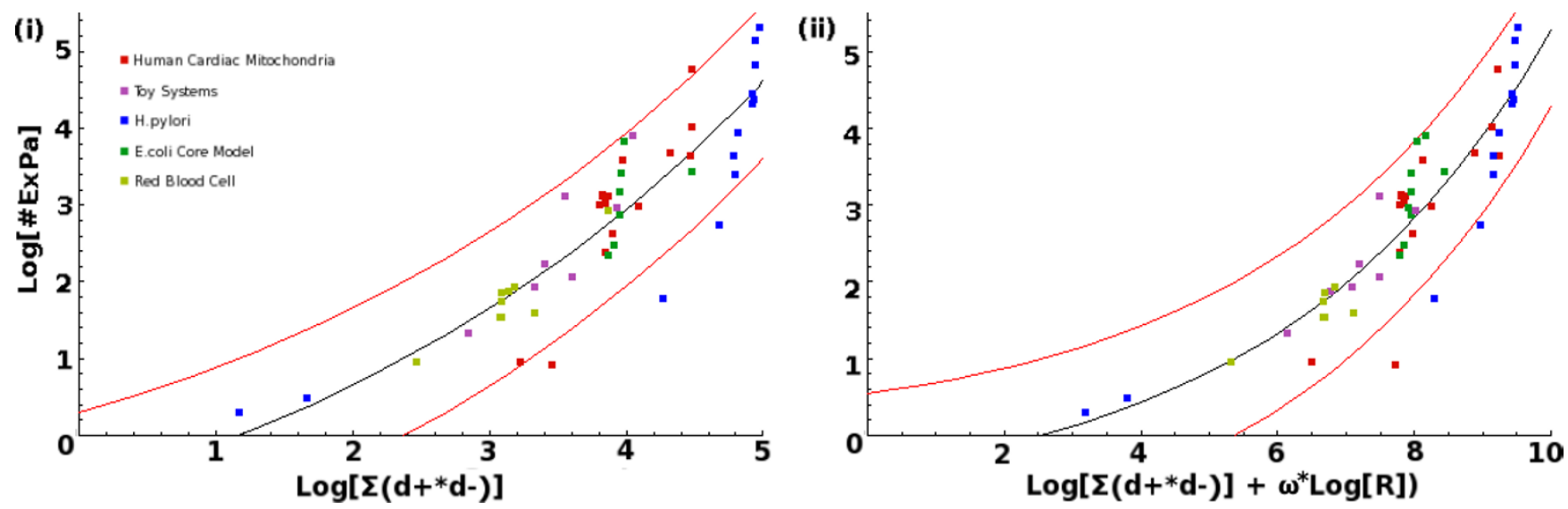

Figure 2

Relationship between the Number of ExPas and Factor $\theta_{2}=\sum_{i=1}^{R} d_{-_{i}} d_{+_{i}}$. Similar to Figure I, it was observed that $\theta_{2}=\sum_{i=1}^{R} d_{-i} d_{+_{i}}$ also had an exponential relation to log $[p]$ (i), which could be improved if scaled by $R^{\omega}$ (ii).

that given by Equation(3) resulted in a better fit, with correlation being 0.900704 and reduced mean absolute- and root-mean-square errors (Table 2). The overall performance of this estimator was evaluated. It was found that the number of ExPas for most of the models (47 out of 52) could be described to within a factor of 10 using the estimating functions, while those that could not tended to be over-estimated (Table 2). The inclusion of the factor $R^{\omega_{2}}$ led to better fits between the estimating function and the training data and reduced average errors.

Estimation Using $\theta_{2}$

Using the second factor, $\theta_{2}$, the estimating functions with and without scaling had the respective forms

$$
f_{1}\left(\theta_{2}\right)=-2.671743+1.927198\left(\sum_{i=1}^{R} d_{-_{i}} d_{+_{i}}\right)^{0.113432}
$$

and

$f_{2}\left(\theta_{2}\right)=-0.971205+0.515711\left(R^{2.124857} \sum_{i=1}^{R} d_{-_{i}} d_{+_{i}}\right)^{0.108329}$.

The relationships between $\log [p]$ and Equations (4) and (5) are displayed in Figure 2. In this case, the Pearson's correlation before the inclusion of $R^{\omega}$ was 0.887057 , and was improved to 0.898332 after scaling.

Table 2: Fit of Training Data to Estimating Functions

\begin{tabular}{|c|c|c|c|c|c|c|c|}
\hline \multirow[b]{2}{*}{ Factor } & \multicolumn{3}{|c|}{ Fit of Factors } & \multicolumn{2}{|c|}{ Over-Estimation } & \multicolumn{2}{|c|}{ Under-Estimation } \\
\hline & Correlation $r$ & m.a.e. & r.m.s. & Max. Value & Members $>1$ & Min. Value & Members $<1$ \\
\hline$\theta_{1}=\sum d_{+} d_{-} c$ & 0.883439 & 0.407565 & 0.539834 & 1.512650 & 5 & -1.138180 & 1 \\
\hline$\theta_{1}=\sum d_{+} d_{-}$ & t0.887057 & 0.406882 & 0.544633 & 1.585230 & 3 & -1.071100 & $\mathrm{I}$ \\
\hline$R^{\omega} \theta_{1}$ & $\ddagger 0.900704$ & 0.383868 & 0.512515 & 1.629060 & 3 & -0.918259 & 0 \\
\hline$R^{\omega} \theta_{1}$ & 0.898332 & 0.381380 & 0.518272 & 1.674300 & 3 & -0.963172 & 0 \\
\hline
\end{tabular}

This table summarizes the statistics describing the relationships between the four different estimations $f_{i}\left(\theta_{j}\right)$ and the 52 data points. The results show that the two factors $\theta_{1}$ and $\theta_{2}$ allowed better estimations when the scaling factor $R^{\omega}$ was included. The top two rows and the bottom two rows of the table, respectively, represent the factors before and after scaling by the factor $R^{\omega}$. The cells with the highest Pearson's correlation, $r$, before and after scaling are emphasized with $\dagger$ and $\ddagger$, respectively. 
Similar to the case for $\theta_{1}$, the errors were reduced after scaling (Table 2). The unscaled estimating function, Equation (4), again, described most of the models (48 out of 52) to within a a factor of 10 with respect to the actual ExPa numbers (Table 2). The inclusion of the scaling factor resulted in less outliers, a better correlation and reduced errors (Table 2).

\section{Performance of Estimations Functions}

The performance of the estimating functions (2-5) was tested using an additional 16 models. These models were reduced but functional models of the central metabolism derived from reconstructed metabolic networks of 3 different organisms, namely H. pylori [3], M. barkeri [16], and $H$. influenzae [1]. All four estimating functions successfully predicted 9 out of the 16 test models (56\%) to within a factor of 10 (Figure 3). For all four estimating functions, the number of ExPas of seven models were overestimated by a factor greater than 10 while none were under-estimated beyond that factor. In particular, the estimating function $f_{2}\left(\theta_{1}\right)$ yielded the smallest error range for all models. The 16 test data points had the highest correlation with $f_{2}\left(\theta_{1}\right)$, as did the 52 data points used for its formulation (Table 3 ). We concluded that the estimating function $f_{2}\left(\theta_{1}\right)$ can typically successfully estimate the number of ExPas of a metabolic network to within a factor of 10 .

\section{Consideration of Other Network Measurements}

During the development of these estimating functions, other factors such as the degrees of exchange metabolites were considered. In the case of exchange metabolites, the correlation of the sum of the degrees of all input metabo-

lites, $D_{i n}=\sum_{i=1}^{\left|M^{-}\right|} d\left(m_{i}^{-}\right)$was found to have a low correlation $(<0.5)$ to the number of extreme pathways, both before and after the data was logged. The correlation for output metabolites, $D_{\text {out }}=\sum_{i=1}^{\left|M^{+}\right|} d\left(m_{i}^{+}\right)$, however, was
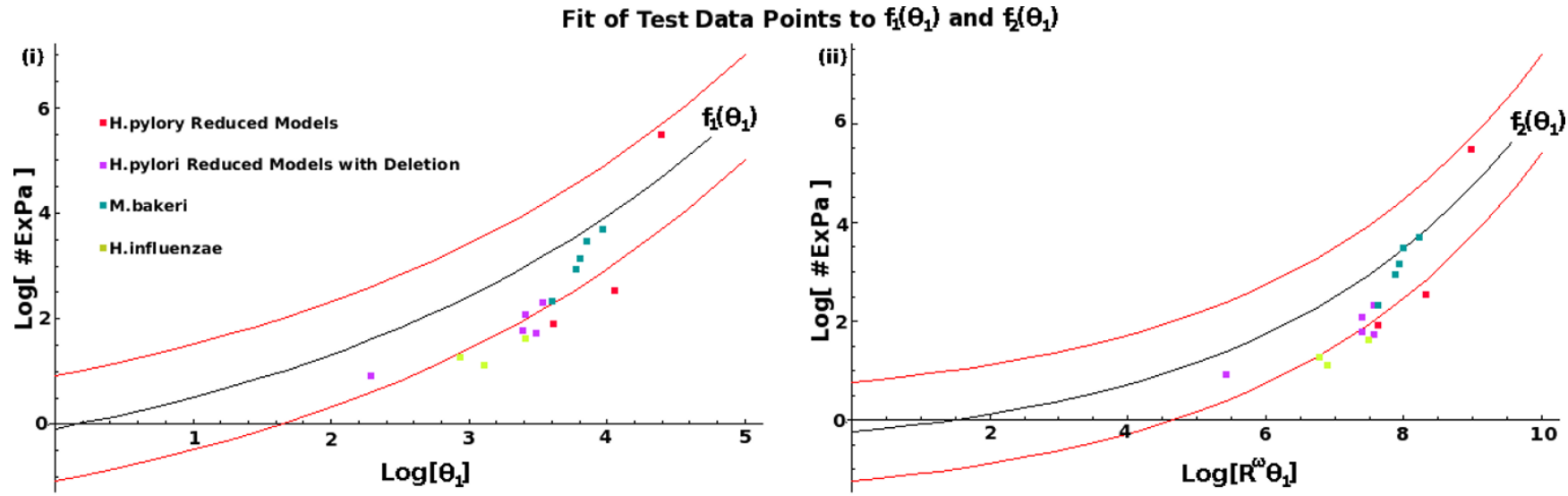

Fit of Test Data Points to $f_{1}\left(\theta_{2}\right)$ and $f_{2}\left(\theta_{2}\right)$
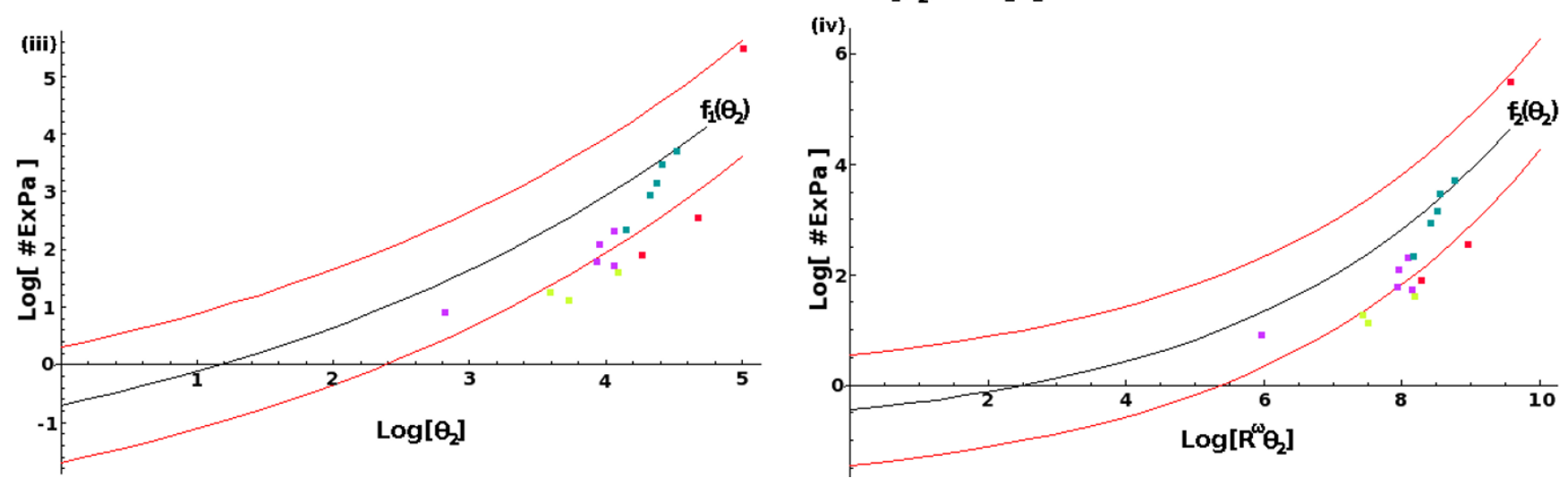

\section{Figure 3}

Comparison of Test Models to the Estimating Functions. Figures displaying the relationships amongst the test data points and the four estimation functions given by equations (2), (3), (4) and (5). These are shown in (i), (ii), (iii) and (iv) respectively. The red lines in each case are given by $f_{i}\left(\theta_{j}\right) \pm \mathrm{I}$ and indicate the boundaries of the regions that are within a factor of 10 of the respective estimations. 
Table 3: Fit of Test Data to Estimating Functions

\begin{tabular}{cccccccc}
\hline & & Fit of Factors & & \multicolumn{2}{c}{ Over-Estimation } & Under-Estimation \\
\hline Factor & Correlation $r$ & m.a.e. & r.m.s. & Max. Value & Members>1 & Min. Value & Members<1 \\
\hline$f_{1}\left(\theta_{1}\right)$ & 0.893058 & 0.956777 & 1.042668 & 1.513670 & 7 & -0.785898 & 0 \\
$f_{2}\left(\theta_{1}\right)$ & $* 0.894445$ & 0.806018 & 0.919502 & 1.353102 & 7 & -0.765522 & 0 \\
$f_{1}\left(\theta_{2}\right)$ & 0.864965 & 0.891715 & 1.004652 & 1.501480 & 7 & -0.851316 \\
$f_{2}\left(\theta_{2}\right)$ & 0.876409 & 0.777881 & 0.897856 & 1.401680 & 7 & -0.808617 & 0 \\
\hline
\end{tabular}

Table detailing the relationships between the 16 test data points and the four estimating functions. Although all four functions failed to predict 7 models to within a factor of 10 of their actual number of ExPas, the function $f_{2}\left(\theta_{1}\right)$ had the least range for over- and under-estimation. Rows representing information of estimating functions using factors $\theta_{1}$ and $\theta_{2}$, respectively, in the top two and bottom two rows. The cell corresponding to the highest correlation, $r$, is emphasized by an asterisk (*).

found to have a high correlation $(>0.5)$ to $p$. Their product, $D_{\text {in }} \cdot D_{\text {out }}$ had correlations of 0.408543 and 0.638261 before and after the data was logged. A similar situation existed for the number of effective reactions $R$, which had a low and high correlation before and after the data was logged. By inspection, a relationship in the bform of Equation (1) could have been established for $R$. However, the range of $R$ did not grow as consistently as $\theta_{1}$ and $\theta_{2}$. As an example, for one $H$. pylori model, 174 effective reactions resulted in 204,412 extreme pathways, whereas for the Mitochondria model with the reaction SUCD3-u10 m removed, 174 effective reactions only produced 4209 ExPas. This then led to the conclusion that $R$ alone was not an appropriate factor of estimation. The fact that the factor $\theta_{1}$ grows consistently with $p$ may have over-shadowed the importance of these factors.

\section{Discussion}

The goal for this study was to produce an estimating function using basic network measurements. Specifically, we aimed to obtain a function such that only a single factor is used for estimation. In principle, it was possible to use a multivariate (polynomial) regression method using a number of the factors described in the section 'Identification of Significant Contributing Factors'. However, the independence assumption upon which this method is based was not applicable as the factors themselves tend to be highly correlated. Furthermore, it would have been difficult to interpret which factors were truly responsible for the increase in $p$, and would probably lead to inaccurate estimations in test models. Here, the most descriptive factor was $\theta_{1}$, which includes the clustering coefficients. The interpretation of the clustering coefficient used in this study is also often used in sociology and biochemical networks (see, for example, $[15,17]$ ). There are other interpretations of the clustering coefficient, such as that described by Soffer et al. [18]. Their definition eliminates degree-correlation biases, thus, quantifying the connectivity amongst the neighbors of a vertex independent to its degree and the degree of its neighbors. It would be interesting to use a similar definition for directed graphs and investigate its effects on ExPa estimation. Additionally, it is possible that other factors may provide a more accurate estimation for the number of ExPas. However, these factors may only be found by detailed analyses of network structures.

The estimating function given by Equation (3) typically estimated the number of ExPas of the test models to within a factor of 10 . In cases where it failed, it did not under-estimate the number of ExPas. The version of the $E$. coli reconstructed network used by Klamt et al. [14] was not elementally- and charge-balanced and has since been replaced by updated versions $[19,20]$. We used a revised version $i J E 660 a$, which was found to be the closest to what they used, and is publicly available $[19,21]$, to compare our method with Klamt's. When the estimating function was applied to this version, assuming that all reactions were active concurrently, $7 \times 10^{12}$ ExPas were estimated with our method, whereas Klamt's method yielded an upper-bound of $5 \times 10^{13}$ after disregarding inactive reactions in the unbalanced and smaller model. Given that iJE660a has 41 more reactions and all the reactions are elementally- and charge-balanced, we are confident that our estimating function can also serve as a conservative upper-bound of the number of ExPas after some adjustments. For larger networks such as the latest published reconstruction of $E$. coli consisting of 904 cited reactions [20], we estimate $3 \times 10^{18}$ ExPas, The Human reconstructed network with 3311 reactions [22] is predicted to have $10^{29}$ ExPas when all reactions were active concurrently.

\section{Conclusion}

In this study, we investigated the possibility of estimating with confidence the number of extreme pathways (ExPa), $p$, for metabolic networks. Our effort concentrated on the use of simple network measurements, namely the incoming and outgoing degrees, $d_{\overline{+}_{i}}$ and the clustering coeffi- 
cients, $c_{i}$, for each of the active reactions, $r_{i} \in R_{\text {eff. }}$ In particular, it was found that $\log [p]$ was correlated to the factors $\theta_{1}=\sum_{i=1}^{R} d_{-_{i}} d_{+_{i}} c_{i}$ and $\theta_{2}=\sum_{i=1}^{R} d_{-_{i}} d_{+_{i}}$ with an exponential relationship. These factors can be calculated quickly and easily, and were found to increase in values consistently with $p$. The resulting estimating functions, in particular that given by Equation (3), typically estimated the number of ExPas to within a factor of 10. Therefore we are confident that these estimating functions are scalable and can be reliably applied to larger networks. These estimating functions will therefore enable researchers to decide whether ExPa analysis is an appropriate investigative tool for their objectives.

The set of extreme pathways is the convex basis used for biochemical characterization of the null-space of the stoichiometric matrix for a biochemical reaction network. ExPa analyses have typically been used to characterize phenotypic states of metabolic networks and identify network redundancy. Beyond these uses, the singular value decomposition of the extreme pathway matrix has been used to identify eigenpathways that are capable of characterizing phenotypic states of a system [23,24]. Nevertheless, applications such as these require ExPas to be calculated prior to any analysis. The number of ExPas is set to increase dramatically with network size and complexity. In particular, with the increase in details of metabolic network reconstructions and the emergence of reconstruction of global transcription/translation networks, new techniques for calculating and analyzing ExPas are much needed. Since the goal of systems biology is to study an organism as a whole, different types of biochemical networks will eventually be combined so that the system can be studied in its entirety. To over-come future computational challenges as well as being equipped with the necessary analytical techniques should become our immediate goal.

\section{Methods}

\section{Basic Concepts and Notations} Hypergraph

We introduce some basic concepts and notations that will assist us in describing the measurements needed. We first note that a metabolic network can be described as a directed-hypergraph, where a node represents a metabolite and an edge a reaction. The stoichiometric matrix, $\mathbf{S}$, can thus be seen as a node-edge incidence matrix. A directed-hypergraph $\mathcal{H}(V, E)$ consists of nodes (vertices) $v \in V$ and edges $e \in E$. Let the matrix $\mathrm{S}$ be the node-edge incident matrix such that $s_{i, j}<0$ if node $v_{i}$ is at the tail of edge $e_{j}, s_{i, j}>0$ if $v_{i}$ is at the head of $e_{j}$ and $s_{i, j}=0$ if $e_{j}$ does not contain $v_{i}$. We define the set of nodes $v$ that are tails (heads) of edge $r$ to be $T(e)(H(e))$. It can easily be seen that $T(e) \cap H(e)=\varnothing$.

\section{Reaction Adjacency and Neighbourhood Matrices $\hat{A}, \hat{\Delta}$}

The adjacency matrix contains information about whether one reaction 'goes into' another. Using the notation introduced in the 'Hypergraph' section, two reactions $r_{i}$ and $r_{j}$ are adjacent if $H\left(r_{i}\right) \cap T\left(r_{j}\right) \neq \varnothing$ or $H\left(r_{j}\right) \cap T\left(r_{i}\right) \neq \varnothing$, that is, the intersection of the set of outputs of reaction $r_{i}$ and the set of inputs for reaction $r_{j}$ is non-empty, or vice versa. In particular, we say $r_{i}$ 'goes into' to $r_{j}$ if $H\left(r_{i}\right) \cap T\left(r_{j}\right) \neq \varnothing$. The adjacency matrix $\hat{A}$ is constructed from the stoichiometric matrix $\mathbf{S}$ by partitioning $\mathbf{S}$ into two digitized components $\hat{\mathbf{S}}_{+}$and $\hat{\mathbf{S}}_{-,}$where $\hat{s}_{+_{i, j}}=1$ if $s_{i, j}>0$ and $\hat{s}_{-i, j}=1$ if $s_{i, j}<0$. Let $\mathbf{S}$ be an $n \times m$ matrix. The adjacency matrix for the reactions is then given by

$\hat{\mathbf{A}}=\left(\hat{a}_{i, j}\right) \in M_{n \times n}(0,1)$ such that $\quad \hat{a}_{i, j}=\left\{\begin{array}{ll}1 & \text { if } i \neq j \\ 0 & \text { otherwise }\end{array}\right.$ and $\hat{\mathbf{s}}_{+_{i}} \cdot \hat{\mathbf{s}}_{-_{j}} \neq 0$,

The elements of the adjacency matrix $\hat{a}_{i, j}=1$ if and only if there exists an metabolite $m_{k}$ such that $s_{k, i}>0$ and $s_{k, j}<$ 0 ; i.e. reaction $r_{i}$ 'goes into' reaction $r_{j}$. In terms of the matrix $\hat{\mathbf{A}}$, two reactions $r_{i}$ and $r_{j}$ are adjacent if either $\hat{a}_{i, j}$ or $\hat{a}_{j, i}$ is non-zero, which is in agreement with the above definition.

Reactions $r_{i}$ and $r_{j}$ are said to be connected if any of $H\left(r_{i}\right) \cap$ $T\left(r_{j}\right), T\left(r_{i}\right) \cap H\left(r_{i}\right), H\left(r_{i}\right) \cap H\left(r_{j}\right)$ or $T\left(r_{i}\right) \cap T\left(r_{j}\right)$ is nonempty. The neighbourhood matrix is given in the form

$\hat{\boldsymbol{\Delta}}=\left(\hat{\delta}_{i, j}\right)$ such that $\hat{\delta}_{i, j}=\left\{\begin{array}{ll}1 & \text { if } i \neq j \\ 0 & \text { otherwise }\end{array}\right.$ and $\hat{\mathbf{s}}_{i} \cdot \hat{\mathbf{s}}_{j} \neq 0$,

where $\hat{s}_{i, j}=1$ if $s_{i, j}$ is non-zero and 0 otherwise, and $\hat{\delta}_{i, j}=1$ iff $r_{i}$ and $r_{j}$ are connected.

\section{Network Measurements}

Effective Number of Reactions $R=\left|R_{\text {eff }}\right|$

For any models of a reconstructed network, redundancy in terms of reactions that are not utilized is often expected. This is due to the fact that, for any specific model, there is a set of reactions that is not used under the specific simulation conditions, and therefore can be removed from the 
network without affecting the model's function. Extreme pathways are classified into 3 types, with Type-I being those that have exchange fluxes across the system boundaries that correspond to non-currency metabolites [25]. Here, we denote the set of reactions that are present in at least one Type-I ExPas by $R=\left|R_{\text {eff }}\right|$. This number can be obtained by optimization techniques such as Flux-Balance Analysis [26] using tools such as SimPheny by Genomatica or FluxAnalyzer [27].

\section{Reaction Connectivity $\mathrm{d}_{ \pm}\left(\mathrm{r}_{\mathrm{i}}\right)=d_{ \pm_{i}}$}

Having identified the set of reactions from a reconstruction that are active in a model, the stoichiometric matrix $\mathbf{S}$ can then be reduced by removing inactive reactions and metabolites. The connectivity (degree) of each active reaction can then be calculated. Since $\mathrm{S}$ can be considered the node-edge incidence matrix for a directed-hypergraph, it is more appropriate to consider the incoming and outgoing metabolites separately. The adjacency and connectivity between each pair of reactions can then be described in terms of the definition given in the section titled 'Reaction Adjacency and Neighbourhood Matrices $\hat{\mathbf{A}}, \hat{\boldsymbol{\Delta}}^{\prime}$. The number of reactions that produce at least one product which is consumed by reaction $r_{i}$ gives rise to the quantity $d_{-i}=d_{i}\left(r_{i}\right)$, and the number of reactions that consume one or more of the products of $r_{i}$ is defined by the quantity $d_{+_{i}}=d_{+}\left(r_{i}\right)$.

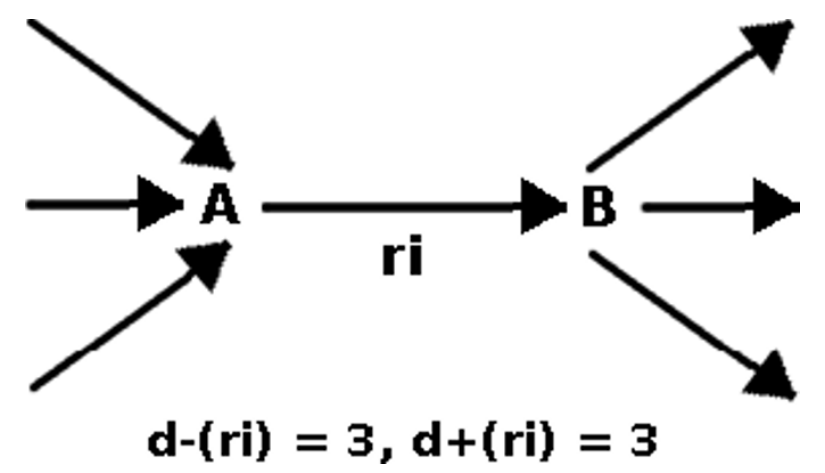

Figure 4

Connectivity of Reactions. Diagram describing different types of connectivities. Reaction $r_{i}$ utilizes metabolite $A$, which is produced by three reactions, and produces metabolite $B$, which is consumed by three reactions. Reaction $r_{i}$ then has an incoming degree of $d_{-}\left(r_{i}\right)=3$ due to metabolite $A$, and outgoing degree of $d_{+}\left(r_{i}\right)=3$.
From Figure 4, it can be seen that the number of possible pathways through a given reaction $r_{i}$ is given by $d_{-_{i}} \cdot d_{t_{i}}$. It is tempting to conclude that the number of pathways calculated is given by $\prod_{i=1}^{R} d_{-_{i}} \cdot d_{+_{i}}$. However, this would be similar to the number derived in [14], which typically over-estimated the number of elementary modes, of which the set of ExPas $\left\{\mathbf{p}_{i}\right\}$ is a subset, by a factor of $6 \times 10^{7}$. Here, we instead looked into the relationship between $p=\left|\left\{\mathbf{p}_{i}\right\}\right|$ and the sum of these terms to avoid such an overestimation.

\section{Reaction Clustering Coefficient $c_{i}=c\left(r_{i}\right)$}

A metabolic network is described by the stoichiometric matrix $\mathbf{S}$. This S-matrix can be seen as a node-edge incidence matrix for a directed hypergraph. However, the clustering coefficient for a hypergraph is not well defined. Since we are interested in how the reactions are connected, we can use the adjacency matrix, $\hat{\mathbf{A}}$, which contains the node-node (reaction-reaction) information of the network, where $\hat{a}_{i, j} \neq 0$ if vertex $v_{i}$ is adjacent to $v_{j}$, i.e., reaction $i$ goes into reaction $j$ (see Figure 5 ). In this configuration, we can then calculate the clustering coefficients for each active reaction using the usual equation

$$
c_{i}=c\left(r_{i}\right)=\frac{\left|\left\{e_{p, q}\right\}\right|}{k_{i}\left(k_{i}-1\right)},
$$

where $k_{i}$ is the number of reactions that $r_{i}$ is connected to, i.e., $k_{i}$ is the number of non-zero elements of the vector $\hat{\delta}_{i}$ of the matrix $\hat{\Delta}$. The set $\left\{e_{p, q}\right\}$ denotes the set of edges going from $r_{p}$ to $r_{q}$ with both $r_{p}$ and $r_{q}$ being connected to $r_{i}$, i.e., $r_{p}$ and $r_{q}$ are connected to $r_{i}$ and are themselves adjacent. The denominator is the number of all such possible edges for a given $r_{i}$. Note that since we are dealing with a directed graph, $e_{p, q}$ is not the same as $e_{q, p}$. Alternative pathways from one set of substrates to one set of products almost always exist in biological networks, especially in metabolic networks. Figure 6 shows that non-zero clustering coefficients are related to alternative routes, and could then be an important factor for determining the number of extreme pathways calculated.

\section{Reconstructed Networks and Simulation Conditions}

Reconstructed metabolic networks of $H$. pylori [3], Human Cardiac Mitochondria [4], the Human Red Blood Cell [5], and the core E. coli [28] network were used in this study. 

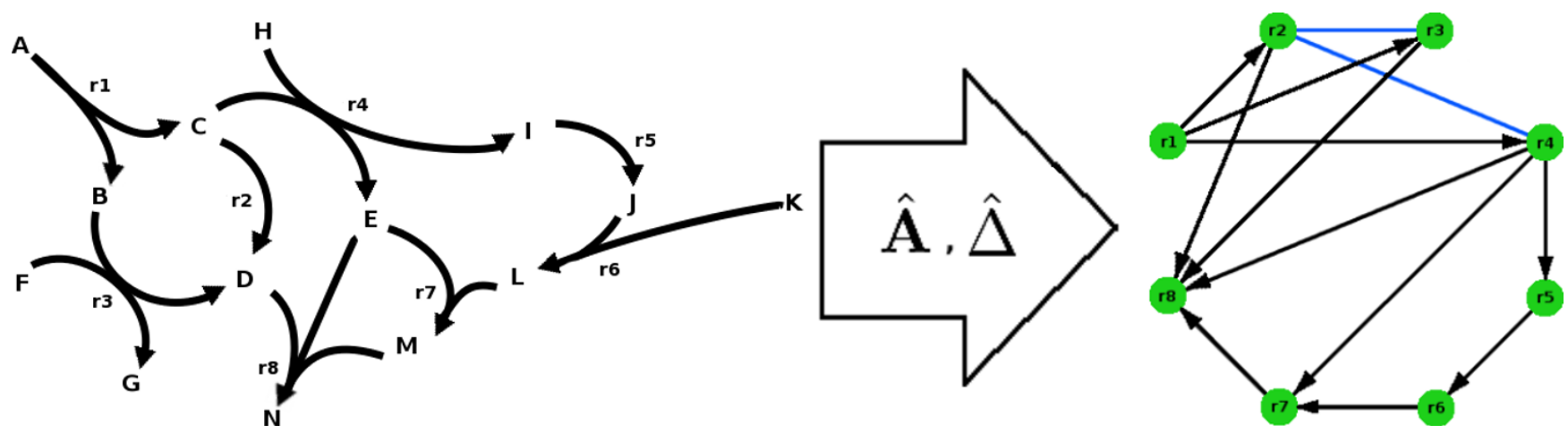

Figure 5

Projection from Directed Hypergraph to One-mode Graph. Projection from directed hypergraph to one-mode graph, where the hyperedges on the left-hand side become the nodes of the the graph on the right-hand side. A thick black arrow in the graph on the right signifies an edge $r_{i}$ is adjacent to $r_{j}$, whereas a thin blue line signifies two edges connected that are not adjacent.

These networks are all mass- and charge-balanced, and were all tested on SimPheny (Genomatica) for the ability to produce biomass constituents. Furthermore, older versions of published, available networks that were used for extreme pathway and flux-balance analysis, which may not be completely balanced, were included in this study. This selection of networks represented a spectrum of complexity. Elementary network measurements, the number of generated models, and the source of each of these networks are detailed in Table 4.

A total of 52 models were used from these networks. Most of these models were used to test for production of products given a specified substrate along with core exchange metabolites. The remaining 9 models included singlereaction deletions and/or the request for specific demand metabolites given a combination of primary substrates.
The specific environmental conditions of these models are listed in Table 5, along with the names of abbreviated metabolites in Table 6.

A total of 16 functional models from 3 different organisms were used to test the validity of estimations formulated in the section 'Single Factor Estimate'. The networks used were 2 reduced versions of $H$. pylori, one consisting of 168 reactions and 170 metabolites, the other with 48 reactions and 65 metabolites. Reduced versions of the networks of $M$. barkeri [16] and $H$. influenzae [1] were also used. These consisted of 84 and 61 reactions and 121 and 83 metabolites, respectively. These networks are similar to the core E. coli model, with each reaction being mass- and charge-balanced. They were also tested for the production of biomass using SimPheny and hence are functional systems.

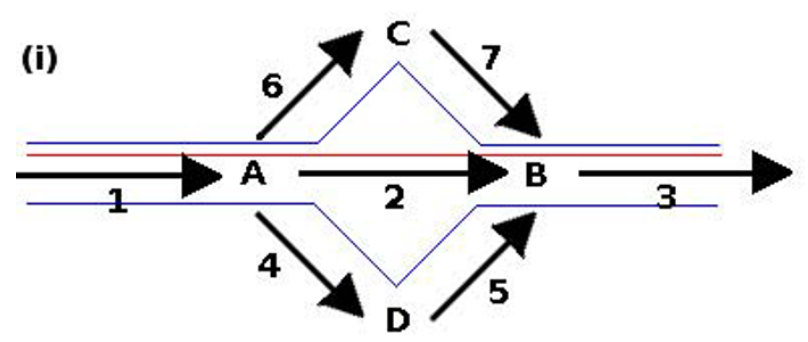

$$
\begin{gathered}
c 1=c 3=1, c 2=8 / 15 \\
c 4=c 5=c 6=c 7=2 / 3
\end{gathered}
$$

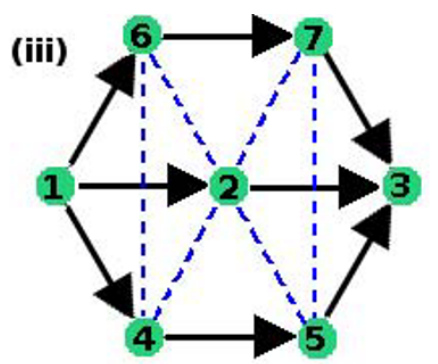

$c 1=c 3=0, c 2=1 / 5$

$c 4=c 5=c 6=c 7=1 / 6$

Figure 6

Relationship between Reactions with Non-zero Clustering-coefficient and Alternative Routes. Diagram showing relationship between non-zero clustering coefficients and alternative pathways. (i) shows three possible routes for a simple system; (ii) is the non-directed representation of this system using the above projection. The system has non-zero clustering coefficients, emphasizing alternative routes are possible; (iii) is the projection conforming to that shown in Figure 5, where nonzero clustering coefficient is found for 5 of the reactions that are involved in the branching points of alterative routes. 
Table 4: Basic Information of Models Used

\begin{tabular}{|c|c|c|c|c|c|}
\hline Network & \# Rxns & Reversible & \# Mets & Models Used & Source \\
\hline H.pylori & 479 & 166 & 485 & 13 & [3] \\
\hline Mitochondria & 200 & 92 & 238 & 16 & [4] \\
\hline Core E.coli & 62 & 35 & 63 & 9 & [28] \\
\hline Central E.coli & 56 & 19 & 62 & 2 & {$[30]$} \\
\hline Toy E.coli & 50 & 37 & 53 & 4 & [3I] \\
\hline Red Blood Cell & 32 & 17 & 39 & 8 & [5] \\
\hline
\end{tabular}

Simple measurements, statistics and sources of the models used in this study.

Table 5: Environmental Conditions of Models. Table detailing the models used for estimation formulation, along with their environmental conditions.

\begin{tabular}{|c|c|c|c|c|c|c|c|}
\hline \multirow[b]{2}{*}{ Organisms } & \multicolumn{2}{|c|}{ Core Metabolites } & \multirow[b]{2}{*}{ Inputs } & \multicolumn{2}{|c|}{ Core Metabolites } & \multirow[b]{2}{*}{ Free } & \multirow[t]{2}{*}{ Specification } \\
\hline & Name & Type & & Outputs & & & \\
\hline \multirow[t]{12}{*}{ H. pylori } & $\mathrm{co} 2$ & Free & $\mathrm{Ac}$ & ac & mal-L & & $\begin{array}{l}\text { Single Input along with core } \\
\text { metabolite, allowing all outputs } \\
\text { (unless specified as input only) }\end{array}$ \\
\hline & fe2 & Free & acac & akg & orn & & \\
\hline & fe3 & Free & ad & asp-L & phe-L & & \\
\hline & h2o & Free & ade & acald & pro-L & & \\
\hline & $\mathrm{h}$ & Free & akg & etoh & pyr & & \\
\hline & nh4 & Free & etoh & fum & ser-L & & \\
\hline & pi & Free & fum & glu-L & succ & & \\
\hline & so4 & Free & gsn & gsn & thr-L & & \\
\hline & 02 & Free & lac & gua & $\operatorname{trp}-\mathrm{L}$ & & \\
\hline & & & mal & $h 2 \cos$ & tyr-L & & \\
\hline & & & $\begin{array}{l}\text { pyr } \\
\text { succ }\end{array}$ & $\begin{array}{l}\text { hxan } \\
\text { lac-L }\end{array}$ & urea & & \\
\hline & & & urea & lys-L & & & \\
\hline \multirow[t]{11}{*}{ Mitochondria } & $\operatorname{co} 2$ & Free & arachd & atp & glu-L & & $\begin{array}{l}\text { Single Input along with core } \\
\text { metabolite, allowing all outputs } \\
\text { (unless specified as input only) }\end{array}$ \\
\hline & $\mathrm{h}$ & Free & bhb & pheme & gly & & \\
\hline & h2o & Free & crvnc & phs-L & glyc & & \\
\hline & $\mathrm{fe} 2$ & Input & glc-D & I2dgr_m & glyc3p & & \\
\hline & 02 & Input & glu-L & acac & hdca & & \\
\hline & pi & Input & hdca & arachd & lac-L & & \\
\hline & urea & Output & lac-L & bhb & ocdca & & \\
\hline & & & ocdc All & coa & ocdcea & & \\
\hline & & & ocdca & crvnc & ocdcya & & \\
\hline & & & ocdcea & cys-L & pheme & & \\
\hline & & & ocdcya & glc-D & ps_m & & \\
\hline \multirow[t]{11}{*}{ Mitochondria } & $\begin{array}{c}\mathrm{co} 2, \mathrm{~h}, \mathrm{~h} 2 \mathrm{fe} 2, \mathrm{fe} 3 \\
\text { o2 urea }\end{array}$ & Free & $\mathrm{acac}$ & atp & & I2dgr_m & $\begin{array}{l}\text { (I) Single K/O of: CYOOm3, } \\
\text { SUCD3-u I Om }\end{array}$ \\
\hline & & Input & arachd & lac-L & & coa & \\
\hline & & Output & Bhb & pheme & & cys-L & \\
\hline & & & crvnc & phs-L & & glu-L & $\begin{array}{l}\text { (2) Individual Request for: atp, phs-L, } \\
\text { pheme }\end{array}$ \\
\hline & & & glc-D & & & gly & \\
\hline & & & glyc & & & Ps_m & \\
\hline & & & glyc3p & & & & \\
\hline & & & hdca & & & & \\
\hline & & & ocdca & & & & \\
\hline & & & ocdcea & & & & \\
\hline & & & ocdcya & & & & \\
\hline E. coli Core & $\mathrm{co} 2$ & Free & $\mathrm{ac}$ & ac & & & $\begin{array}{l}\text { Single Input along with core } \\
\text { metabolite, allowing all outputs } \\
\text { (unless specified as input only) }\end{array}$ \\
\hline
\end{tabular}


Table 5: Environmental Conditions of Models. Table detailing the models used for estimation formulation, along with their environmental conditions. (Continued)

\begin{tabular}{|c|c|c|c|c|c|c|c|}
\hline & $\mathrm{h}$ & Free & akg & akg & & & \\
\hline & h2o & Free & etoh & etoh & & & \\
\hline & 02 & Input & for & for & & & \\
\hline & pi & Input & fum & fum & & & \\
\hline & & & glc-D & lac-D & & & \\
\hline & & & lac-D & pyr & & & \\
\hline & & & $\begin{array}{l}\text { pyr } \\
\text { succ }\end{array}$ & succ & & & \\
\hline \multirow[t]{11}{*}{ RBC } & $\begin{array}{l}\text { adp, atp, co2, h, } \\
\text { h2o, nad, nadh } \\
\text { nadp, nadph, nh3, pi }\end{array}$ & Free & ade & 23dpg & ino & & $\begin{array}{l}\text { (I) Single Input along with core } \\
\text { metabolite, allowing all outputs } \\
\text { (unless specified as input only) }\end{array}$ \\
\hline & & Free & ado & ade & lac & & \\
\hline & & Free & glc & ado & pyr & & \\
\hline & & & hx & glc & & & \\
\hline & & & ino & hx & & & \\
\hline & & & lac & & & & \\
\hline & & & Pyr & & & & \\
\hline & & & glc & $23 \mathrm{dpg}$ & & ade & (2) Multiple Inputs \\
\hline & & & & hx & & ado & \\
\hline & & & & & & ino & \\
\hline & & & & & & $\begin{array}{l}\text { Pyr } \\
\text { ino }\end{array}$ & \\
\hline \multirow[t]{14}{*}{ Central E. coli } & adp, atp, coa, co2, & Free & glycogen & $2 \mathrm{dmmq} \mid 8$ & mqn8 & & (I) Glycogen as Primary Input \\
\hline & & Free & & $2 \mathrm{dmmg} 8$ & $\mathrm{mgl8}$ & & \\
\hline & & Free & & $3 p g$ & oaa & & \\
\hline & & & & akg & pep & & \\
\hline & & & & amp & pyr & & \\
\hline & & & & e4p & q8 & & \\
\hline & & & & fad & q8h2 & & \\
\hline & & & & fadh2 & r5p & & \\
\hline & & & fad & $2 \mathrm{dmmq} 8$ & pyr & & (2) A different set of Primary Input \\
\hline & & & q8 & fadh2 & pep & & \\
\hline & & & $2 \mathrm{dmmql} 8$ & q8h2 & e4p & & \\
\hline & & & & $\mathrm{amp}$ & r5p & & \\
\hline & & & & oaa & $3 p g$ & & \\
\hline & & & & akg & & & \\
\hline
\end{tabular}

Table 6: External Metabolites Abbreviation

\begin{tabular}{|c|c|c|c|}
\hline \multicolumn{4}{|c|}{ Exchange Metabolite Abbreviation } \\
\hline I2dgr_m & I,2-Diacylglycerol & hx & Hypoxanthine \\
\hline $23 \mathrm{dpg}$ & 2,3-Phospho-D-glyceroyl phosphate & hxan & Hypoxanthine \\
\hline $2 \mathrm{dmmq} 8$ & 2-Demethylmenaquinone 8 & ile-L & L-Isoleucine \\
\hline $2 \mathrm{dmmql8}$ & 2-Demethylmenaquinol 8 & ino & Inosine \\
\hline $3 p g$ & 3-Phospho-D-glycerate & lac & Lactate \\
\hline ac & Acetate & lac-L & L-Lactate \\
\hline acac & Acetoacetate & leu-L & L-Leucine \\
\hline acald & Acetaldehyde & lys-L & L-Lysine \\
\hline ad & Acetamide & mal & Malate \\
\hline ade & Adenine & mal-L & L-Malate \\
\hline ado & Adenosine & meoh & Methanol \\
\hline adp & ADP & $\mathrm{mql8}$ & Menaquinol 8 \\
\hline akg & 2-Oxoglutarate & mqn8 & Menaquinone 8 \\
\hline ala-L & L-Alanine & nad & Nicotinamide adenine dinucleotide \\
\hline ala-S & S-Alanine & nadh & Nicotinamide adenine dinucleotide - reduced \\
\hline amp & AMP & nadp & Nicotinamide adenine dinucleotide phosphate \\
\hline arachd & Arachidonic Acid (C20:4) & nadph & Nicotinamide adenine dinucleotide phosphate - reduced \\
\hline asp-L & L-Asparagine & nh3 & Ammonium \\
\hline
\end{tabular}


Table 6: External Metabolites Abbreviation (Continued)

\begin{tabular}{|c|c|c|c|}
\hline atp & ATP & nh4 & Ammonium lon \\
\hline bhb & (2)-3-Hydroxybuanoate & 02 & $\mathrm{O} 2$ \\
\hline $\operatorname{ch} 4$ & Methan & oaa & Oxaloacetate \\
\hline $\operatorname{co2}$ & $\mathrm{CO} 2$ & ocdc All & octadecanoate, octadecenoate, octadecynoate \\
\hline coa & Coenzyme A & ocdca & octadecanoate \\
\hline crvnc & Cervonic Acid (C22:6, n-3) & ocdcea & octadecenoate \\
\hline cys-L & L-Cysteine & ocdcya & octadecynoate \\
\hline e4p & D-Erythrose 4-phosphate & orn & Ornithine \\
\hline etoh & Ethanol & pep & Phosphoenolpyruvate \\
\hline fad & Flavin adenine dinucleotide & phe-L & L-Phenylalanine \\
\hline fadh2 & Flavin adenine dinucleotide (reduced form) & pheme & Protoheme \\
\hline $\mathrm{fe} 2$ & Iron (II) & phs-L & Phospholipid \\
\hline fe3 & Iron (III) & pi & Phosphate \\
\hline for & Formate & ppi & Diphosphate \\
\hline fum & Fumarate & pro-L & L-Proline \\
\hline glc & Glucose & ps_m & Phosphatidylserine \\
\hline glc-D & D-Glucose & pyr & Pyruvate \\
\hline glu-L & L-Glutamate & q8 & Ubiquinone-8 \\
\hline gly & Glycine & $98 \mathrm{~h} 2$ & Ubiquinol-8 \\
\hline glyc & Glycerol & r5p & alpha-D-Ribose 5-phosphate \\
\hline glyc3p & Glycerol 3-phosphate & ser-L & L-Serine \\
\hline gsn & Guanosine & so4 & Sulfate \\
\hline gua & Guanine & succ & Succinate \\
\hline $\mathrm{h}$ & $\mathrm{H}+$ & thr-L & L-Threonine \\
\hline h2 & $\mathrm{H} 2$ & trp-L & L-Tryptophan \\
\hline $\mathrm{h} 2 \mathrm{co} 3$ & carbonic acid & tyr-L & L-Tyrosine \\
\hline h2o & $\mathrm{H} 2 \mathrm{O}$ & urea & Urea \\
\hline hdca & Hexadecanoate (n-Cl6:0) & val-L & L-Valine \\
\hline
\end{tabular}

Abbreviations of all external metabolite found in the 68 models used in this study.

The number of data points may seem unorthodox Although in theory it was possible to automatically generate networks of different input/output combinations, leading to a larger number of training and validation data points, in practice, only a few of such combinations would have resulted in models that produce non-Type-III extreme pathways as well as biomass constituents. For the validation stage, models producing biomass constituents as well as non-Type-III pathways that could be calculated quickly were desired. By drastically reducing networks, it was difficult to construct models that maintained biomass production.

In the case of $H$. pylori, three models were produced using the larger network in its entirety. Since the computation of ExPas is a time-consuming process, a smaller network was created to facilitate this process. This smaller network was subjected to random reaction-deletion while ensuring that the subsequent modified models could still produce equal amount of biomass. Five such models with random deletion were produced for this study. In addition, 5 models for $M$. bakeri and 3 for $H$. influenzae were generated in a similar fashion so that ExPa computation could be done within a reasonable computational time and effort. These are listed in Table 7.

\section{Calculation of Extreme Pathways and Network Measurements}

The extreme pathways of all models were computed using an implementation of the algorithm given in [29]. This implementation includes the $\mathrm{C}++$ STL and the number theory library NTL. Algorithms for calculating the greatest common factor of a set of integers of arbitrary size and for sparse-matrix operations were also implemented. Network properties, including incoming and outgoing degrees and clustering coefficients of reactions, were calculated using a $\mathrm{C}++$ implementation of the methods described in 'Basic Concepts and Notations' and 'Network Measurements' using sparse-matrix algorithms and bitwise operations.

\section{Correlation Coeffcients}

Both Pearson's product-moment and Spearman's rank correlation coefficients were used as a guide to help identify important factors that contribute to the number of extreme pathways. The former is defined as

$$
r=\frac{n \sum_{i=1}^{n} x_{i} y_{i}-\sum_{i=1}^{n} x_{i} \sum_{i=1}^{n} y_{i}}{\sqrt{n \sum_{i=1}^{n} x_{i}^{2}-\left(\sum_{i=1}^{n} x_{i}\right)^{2}} \sqrt{n \sum_{i=1}^{n} y_{i}^{2}-\left(\sum_{i=1}^{n} y_{i}\right)^{2}}}
$$


Table 7: Environmental Conditions of Test Models

\begin{tabular}{|c|c|c|c|}
\hline Organism & \#Rxn & \#Met & External Metabolites \\
\hline M. barkeri & 84 & 121 & ac, ala-L, alac-S, ch4, co2, cys-L, gly, h, h2 h2o, ile-L, leu-L, meoh, pi, pyr, val-L \\
\hline H. influenzae & 61 & 83 & ac, akg, co2, for, fum, glc-D, h, hxan nh4, mal-L, pi, pyr \\
\hline H. pylori & 48 & 65 & acald, akg, co2, etoh, for, fum, glc-D, h h2co3, lac-L, mal-L, o2, pi \\
\hline H. pylori & 168 & 170 & $\begin{array}{l}\text { ac, acald, akg, asp-L, co2, etoh, fum, glc-D, glu-L, h, h2co3, h2o, lac-L, lys-L, mal-L, nh4, } \\
\text { o2, phe-L, pi, pyr, ser-L, succ, thr-L, trp-L, tyr-L, urea }\end{array}$ \\
\hline
\end{tabular}

Simple network measurements and the lists of external metabolites for the networks used to test the capability of estimations developed in the section 'Single Factor Estimate'.

for a series of $n$ measurements $x_{i}$ and $y_{i}$. It was used in this study as a guide to detect linear relationships amongst the data and estimating functions. The non-parametric correlation coefficient used in this study is defined as

$$
\rho=1-\frac{6 \sum_{i=1}^{n} D_{i}^{2}}{n\left(n^{2}-1\right)},
$$

where $D_{i}$ is the difference in the ranks of the corresponding values of the $n$ pairs $\left(x_{i}, y_{i}\right)$. This was used to decide whether a factor increased monotonically with the number of ExPas $p$.

\section{Authors' contributions}

MY and BØP designed the study. MY performed and developed the programs used for the calculation of extreme pathways and network measurements, and analyzed and interpreted the data. IT provided all data of reconstructed models used in this study. MY drafted the manuscript while IT and BØР provided critical edits and important intellectual content. MY, IT \& BØP have read and approved the final version of this manuscript.

\section{Acknowledgements}

This work was funded by National Institute of General Medical Sciences (NIGMS, grant number GM68837). BØP serves on the Scientific Advisory Board of Genomatica Inc. The authors would like to thank Marc Abrams for critical reading of this manuscript.

\section{References}

I. Schilling $\mathrm{CH}$, Palsson $\mathrm{B} \varnothing$ : Assessment of the Metabolic Capabilities of Haemophilus influenzae Rd through a Genome-scale Pathway Analysis. Journal of Theoretical Biology 2000, 203:249-283.

2. Stucki JW: Chromokinetics of Metabolic Pathways. European Journal of Biochemistry 2004, 27 I:2745-2754.

3. Thiele I, Vo TD, Price ND, Palsson BØ: Expanded Metabolic Reconstruction of Helicobacter pylori (iIT34 I GSM/GPR): an In Silico Genome-Scale Characterization of Single- and Double-Deletion Mutants. Journal of Bacteriology 2005, I 87(16):58|8-5830.

4. Vo TD, Greenberg HJ, Palsson BØ: Reconstruction and Functional Characterization of the human Mitochondria Metabolic Network Based on Proteomic and Biochemical Data. Journal of Biological Chemistry 2004, 279(38):39532-39540.

5. Wiback $S$ J, Palsson $B \varnothing$ : Extreme pathway Analysis of Human Red Blood Cell Metabolism. Biophysical Journal 2002, 83:808-8I8.

6. Gianchandani EP, Papin JA, Price ND, Joyce AR, Palsson BØ: Matrix Formalism to Describe Functional States of Transcriptional Regulatory Systems. PLoS Compuational Biology 2006, 2(8):eI0I.
7. Xiong MM, Xiong JZ, Xiong H: Network-based RegulatoryPathway Analysis. Bioinformatics 2004, 20(13):2056-2066.

8. Papin JA, Palsson BØ: The JAK-STAT Signaling Network in the Human B-Cell: An Extreme Signaling Pathway Analysis. Biophysical Journal 2004, 87:37-46.

9. Papin JA, Price ND, Edwards JS, Palsson BØ: The Genome-Scale Metabolic Extreme Pathway Structure in Haemophilus influenzae Shows Significant Network Redundancy. Journal of Theoretical Biology 2002, 215:67-82.

10. Price ND, Papin JA, Palsson BØ: Determination of Redundancy and Systems Properties of the Metabolic Network of Helicobacter pylori Using Genome-Scale Extreme Pathway Analysis. Genome Research 2002, 12:760-769.

II. Schuster S, Fell DA, Dandekar T: A general definition of metabolic pathways useful for systematic organization and analysis of complex metabolic networks. Nature Biotechnology 2000, 18:326-332.

12. Papin JA, Price ND, Wiback SJ, Fell DA, Palsson BØ: Metabolic Pathways in the Post-Genome Era. Trends in Biochemical Sciences 2003, 28(5):250-258.

13. Cormen TH, Leiserson CE, Rivest RL, Stein C: Introduction to Algorithms second edition. MIT Press and McGraw-Hill; 200I.

14. Klamt S, Stelling J: Combinatorial Complexity of Pathway Analysis in Metabolic Networks. Molecular Biology Reports 2002, 29:233-236.

15. Barabási AL, Oltvai ZN: Network Biology: Understanding the Cell's Functional Organization. Nature Review 2004, 5: I0 I-I I3.

16. Feist AM, Scholten JCV, Palsson B $\varnothing$, Brockman FJ, Ideker T: Modeling Methanogenesis with a Genome-Scale Metabolic Reconstruction of Methanosarcina barkeri. Molecular Systems Biology 2006, 2:.

17. Heyman K: Making Connections. Science 2006, 3 I 3:604-606.

18. Soffer SN, Vázquez A: Network Clustering Coefficient without Degree-Correlation Biases. Physical Review 2005, 71:057101 $-057104$.

19. Edwards JS, Palsson BØ: The Escherichia coli MGI655 in silico metabolic genotype: its definition, characteristics, and capabilities. Proc Natl Acad Sci USA 2000, 97:5528-5533.

20. Reed JL, Vo TD, Schilling $C H$, Palsson $B \varnothing$ : An expanded genomescale model of Escherichia coli K-I2 (iJR904 GSM/GPR). Genome Biology 2003, 4(9):R54.I-R54. 12.

21. Reaction list of E. coli iJR904 [http://systemsbiology.ucsd.edu/ organisms/ecoli/ecoli reactions.html]

22. Duarte NC, Becker SA, Jamshidi N, I T, Mo ML, Vo TD, Srivas R, Palsson $B \varnothing$ : Global reconstruction of the human metabolic network based on genomic and bibliomic data. PNAS 2007, 104:1777-1782

23. Price ND, Reed JL, Papin JA, Palsson BØ: Analysis of Metabolic Capabilities using Singular Value Decomposition of Extreme Pathway Matrices. Biophysical Journal 2003, 84:794-804.

24. Price ND, Reed JL, Papin JA, Wiback SJ, Palsson BØ: Networkbased Analysis of Metabolic Regulation in the Human Red Blood Cell. Journal of Theoretical Biology 2003, 225:185-194.

25. Price ND, Famili I, Beard DA, Palsson BØ: Extreme Pathways and Kirchoff's Second Law. Biophysical Journal 2002, 83:2879-2882.

26. Schilling CH, Edwards JS, Palsson BØ: Towards Metabolic Phenomics: Analysis of Genomic Data Using Flux Balances. Biotechnology Progress 1999, I 5(3):288-295.

27. Klamt S, Stelling J, Ginkel M, Gilles ED: FluxAnalyzer: exploring structure, pathways and flux distribution in metabolic net- 
works on interactive flux maps. Bioinformatics 2003, 1 9(2):26I-269.

28. Palsson BØ: Systems Biology: Properties of Reconstructed Networks Cambridge University Press; 2006.

29. Schilling CH, Letscher DL, Palsson BØ: Theory for the Systemic Definition of Metabolic Pathways and their use in Interpreting Metabolic Function from a Pathway-Oriented Perspective. Journal of Theoretical Biology 2000, 203:229-248.

30. Bell SL, Palsson BØ: ExPA: A Program for Calculating Extreme Pathways in Biochemical Reaction Networks. Journal of Bacteriology 2004, 187(16):5818-5830.

31. Schilling $C H$, Edwards $J$, Letscher DL, Palsson $B \varnothing$ : Combining Pathway Analysis with Flux Balance Analysis for the Comprehensive Study of Metabolic Systems. Biotechnology and Bioengineering 200I, 7 I (4):286-306.

Publish with Biomed Central and every scientist can read your work free of charge

"BioMed Central will be the most significant development for disseminating the results of biomedical research in our lifetime. "

Sir Paul Nurse, Cancer Research UK

Your research papers will be:

- available free of charge to the entire biomedical community

- peer reviewed and published immediately upon acceptance

- cited in PubMed and archived on PubMed Central

- yours - you keep the copyright

Submit your manuscript here:

http://www.biomedcentral.com/info/publishing_adv.asp
BioMedcentral 